든 SCHOOL of GRADUATE STUDIES

EAST TENNESSEE STATE UNIVERSITY
East Tennessee State University Digital Commons@ East Tennessee State University

Electronic Theses and Dissertations

Student Works

$5-2019$

\title{
Perfect Double Roman Domination of Trees
}

Ayotunde Egunjobi

East Tennessee State University

Follow this and additional works at: https://dc.etsu.edu/etd

Part of the Discrete Mathematics and Combinatorics Commons

\section{Recommended Citation}

Egunjobi, Ayotunde, "Perfect Double Roman Domination of Trees" (2019). Electronic Theses and Dissertations. Paper 3576. https://dc.etsu.edu/etd/3576

This Thesis - Open Access is brought to you for free and open access by the Student Works at Digital Commons @ East Tennessee State University. It has been accepted for inclusion in Electronic Theses and Dissertations by an authorized administrator of Digital Commons @ East Tennessee State

University. For more information, please contact digilib@etsu.edu. 
Perfect Double Roman Domination of Trees

A thesis

presented to

the faculty of the Department of Mathematics

East Tennessee State University

In partial fulfillment

of the requirements for the degree

Master of Science in Mathematical Sciences

by

Ayotunde Tolulope Egunjobi

May 2019

Teresa W. Haynes, Ph.D., Chair

Robert A. Beeler, Ph.D.

Rodney Keaton, Ph.D.

Keywords: Roman domination, perfect Roman domination, double Roman domination, perfect double Roman domination 


\section{ABSTRACT \\ Perfect Double Roman Domination of Trees \\ by \\ Ayotunde Tolulope Egunjobi}

A perfect double Roman dominating function of a graph $G$, abbreviated PDRDfunction, is a function $f: V(G) \rightarrow\{0,1,2,3\}$ satisfying the conditions that if $f(u)=$ 0 , then $u$ is adjacent to exactly two vertices in $V_{2}$ and no vertex in $V_{3}$ or exactly one vertex in $V_{3}$ and no vertex in $V_{2}$ and if $f(u)=1$, then $u$ is adjacent to exactly one vertex in $V_{2}$ and no vertex in $V_{3}$. The perfect double Roman domination number, denoted $\gamma_{d R}^{p}(G)$, is the minimum weight of a PDRD-function of $G$. We prove that if $T$ is a tree of order $n \geq 3$, then $\gamma_{d R}^{p}(T) \leq \frac{3}{2} n$. 
(C)Ayotunde Tolulope Egunjobi, 2019

All Rights Reserved 


\section{DEDICATION}

With love, I would like to dedicate this thesis to God and my family in appreciation of their love, encouragement and support during my graduate program. 


\section{ACKNOWLEDGMENTS}

I would like to thank God for helping through the course of my study in ETSU. Also, I would like to thank the Chair of my Advisory Committee Dr. Teresa Haynes, for her support and advice through the course of my thesis work. She provided me with useful ideas on how to go about my research problem which really helped me complete it in due time. I would also like to acknowledge the efforts of my thesis committee members, Dr. Robert Beeler and Dr. Rodney Keaton.

Finally, I would like to thank other ETSU Math Department faculty members and my colleagues currently in the graduate program. The last two years have been amazing for me and I am grateful for the support I received throughout my study here in ETSU. 
TABLE OF CONTENTS

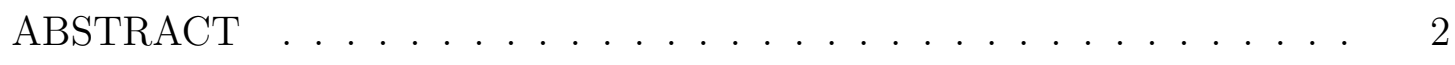

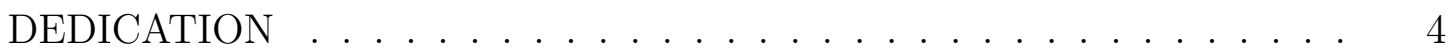

ACKNOWLEDGMENTS ......................... 5

LIST OF FIGURES . . . . . . . . . . . . . . . . . . . . 7

$1 \quad$ INTRODUCTION . . . . . . . . . . . . . . . . . . 8

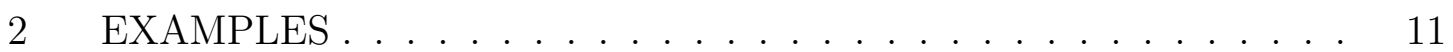

$3 \quad$ LITERATURE SURVEY . . . . . . . . . . . . . . . . . . . 13

3.1 Double Roman Domination ............. 13

3.2 Perfect Roman Domination . . . . . . . . . . . . . 14

$4 \quad$ BASIC RESULTS . . . . . . . . . . . . . . . . . . 16

$5 \quad$ MAIN RESULTS . . . . . . . . . . . . . . . . . . . . . 21

6 CONCLUDING REMARKS . . . . . . . . . . . . . . . 31

BIBLIOGRAPHY . . . . . . . . . . . . . . . . 32

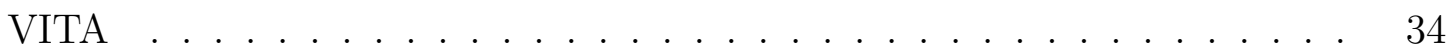




\section{LIST OF FIGURES}

$1 \quad$ Graph $G \ldots \ldots \ldots \ldots$. . . . . . . . . . . . . . . 11

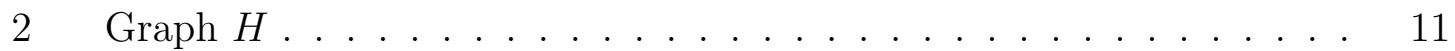

$3 \quad$ A tree $T \in \mathcal{F} \ldots \ldots \ldots \ldots \ldots$

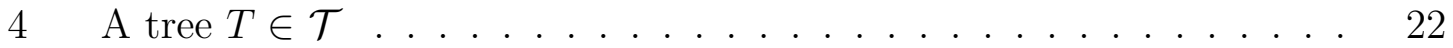

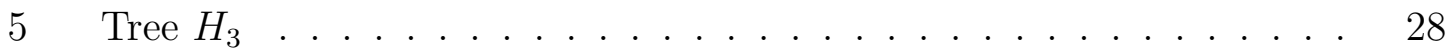

$6 \quad$ A tree $T$ with $\gamma_{d R}^{p}(T) \approx 9 n / 7 \ldots \ldots . \ldots . \ldots . \ldots 30$ 


\section{INTRODUCTION}

Roman domination was motivated by the defense strategies used to defend the Roman Empire during the reign of Emperor Constantine the Great, 274-337 AD. Roman domination in graphs was first introduced by Cockayne, Dreyer and Hedetniemi in 2004 in [6]. It has been studied in the last decade and over 100 papers have been published on various aspects of Roman domination in graphs including Roman domination, independent Roman domination, weak Roman domination, double Roman domination and perfect Roman domination. We need to define some terminology and notation for the purpose of this thesis.

Let $G$ be a finite, simple, and undirected graph with vertex set $V=V(G)$ and edge set $E=E(G)$. The order of $G$, denoted $|V(G)|=n$, is the number of vertices in $G$. The size of $G$, denoted $|E(G)|=m$, is the number of edges in $G$. For any two vertices $x, y \in V(G), x$ and $y$ are adjacent if the edge $x y \in E(G)$. The open neighborhood of $v$ in $V$ is the set $N(v)=\{u \in V: u v \in E\}$ and the closed neighborhood of $v \in V$ is the set $N[v]=N(v) \cup\{v\}$. The open neighborhood of a set $D \subseteq V$ is the set $N(D)=\cup_{v \in D} N(v)$, and the closed neighborhood of a set $D$ is the set $N[D]=N(D) \cup D$. The degree of $v$ is the cardinality of the open neighborhood of $v$, or $\operatorname{deg}_{G}(v)=|N(v)|$. A vertex with exactly one neighbor is called a leaf and its neighbor is a support vertex. A support vertex with two or more leaf neighbors is called a strong support vertex. The independence number of $G$, denoted $\alpha(G)$, is the cardinality of the largest independent set of vertices in $G$. A path $P_{n}$ is a graph of order $n$ and size $n-1$ with vertices denoted $v_{1}, v_{2}, \ldots, v_{n}$ and edges $v_{i} v_{i+1}$ for $i=1,2, \ldots, n-1$. A cycle $C_{n}$ is a graph of order $n$ and size $n$ with vertices denoted 
$v_{1}, v_{2}, \ldots, v_{n}$ and edges $v_{1} v_{n}, v_{i} v_{i+1}$ for $i=1,2, \ldots, n-1$. A subgraph $H$ of a graph $G$ is a graph contained in $G$, i.e., $V(H) \subseteq V(G)$ and $E(H) \subseteq E(G)$.

A dominating set of a graph $G$ is a nonempty subset $D$ of the vertex set $V$ such that for each $u \in V-D$, there exists a $v \in D$ adjacent to $u$. Equivalently, a subset $D$ of $V$ is a dominating set if for each $v \in V,|N[v] \cap D| \geq 1$. A dominating set having the smallest cardinality among all dominating sets in a given graph is called a minimum dominating set. The cardinality of a minimum dominating set in graph $G$ is called the domination number of $G$ and is denoted $\gamma(G)$.

Let $f: V \rightarrow\{0,1,2\}$ be a function having the property that for every vertex $v \in V$ with $f(v)=0$, there exists a neighbor $u \in N(v)$ with $f(u)=2$. Such a function is called a Roman dominating function $(R D F)$. The weight of a RDF is the sum $f(v)=\sum_{v \in V} f(v)$. The minimum weight among all RDF's of $G$ is called the Roman domination number of $G$ and is denoted $\gamma_{R}(G)$. A RDF of $G$ with weight $\gamma_{R}(G)$ is called a $\gamma_{R^{-}}$function of $G$. A perfect Roman dominating function on a graph $G$ introduced in [12] is a function $f: V \rightarrow\{0,1,2\}$ satisfying the condition that every vertex $u \in V$ with $f(u)=0$ is adjacent to exactly one vertex $v$ for which $f(v)=2$. A function $f: V \rightarrow\{0,1,2,3\}$ is a double Roman dominating function on a graph $G$ if the following conditions are met. Let $V_{i}$ denote the set of vertices assigned weight $i$ by function $f$.

1. If $f(v)=0$, then vertex $v$ must have at least two neighbors in $V_{2}$ or one neighbor in $V_{3}$.

2. If $f(v)=1$, then vertex $v$ must have at least one neighbor in $V_{2} \cup V_{3}$. 
Now, we propose perfect double Roman domination. A perfect double Roman dominating function of a graph $G$, abbreviated PDRD-function, is a function $f$ : $V(G) \rightarrow\{0,1,2,3\}$ satisfying the following conditions:

1. If $f(u)=0$, then $u$ is adjacent to exactly two vertices in $V_{2}$ and no vertex in $V_{3}$ or exactly one vertex in $V_{3}$ and no vertex in $V_{2}$.

2. If $f(u)=1$, then $u$ is adjacent to exactly one vertex in $V_{2}$ and no vertex in $V_{3}$.

The perfect double Roman domination number, denoted $\gamma_{d R}^{p}(G)$, is the minimum weight among all PDRD-functions of $G$. That is, $\gamma_{d R}^{p}(G)=\min \{w(f): f$ is a PDRD-function in $G\}$.

A PDRD-function of $G$ with weight $\gamma_{d R}^{p}(G)$ is called a $\gamma_{d R^{-}}^{p}$ function of $G$. Note that a PDRD-function always exist for all graphs by assigning weight 2 to every vertex in the graph. Hence, a graph $G$ of order $n$ satisfies $\gamma_{d R}^{p}(G) \leq 2 n$. 


\section{EXAMPLES}

In this section, we consider the following examples.

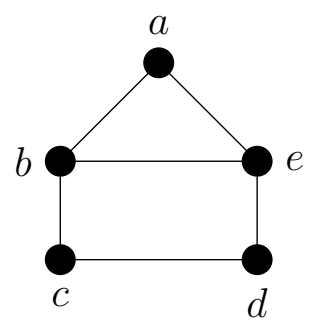

Figure 1: Graph $G$

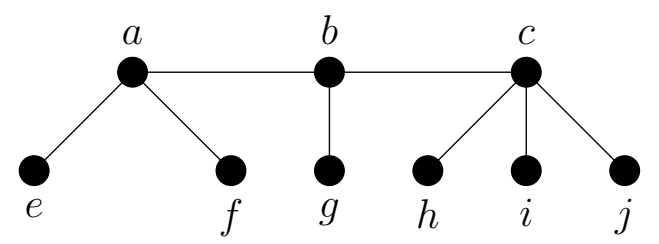

Figure 2: Graph $H$

For the graph $G$ in Figure $1, \gamma_{d R}(G)=\gamma_{d R}^{p}(G)=5$. This is attained by assigning weight 2 to each of $c$ and $e$, weight 1 to $a$ and weight 0 to $b$ and $d$.

For the graph $H$ in Figure 2, $\gamma_{d R}(H)=8$. This is attained by assigning weight 3 to each of $a$ and $c$, weight 2 to $g$ and weight 0 to all other vertices. But this is not a PDRD-function of $H$ as vertex $b$ is over-dominated.

First, note that $\gamma_{d R}^{p}(H) \leq 9$, since the function assigning weight 3 to each $a, b$, and $c$, weight 0 to all other vertices in $H$ is a PDRD-function of $H$. We claim that $\gamma_{d R}^{p}(H) \geq 9$. Suppose to the contrary that $\gamma_{d R}^{p}(H) \leq 8$. Note that any $\gamma_{d R^{-}}^{p}$-function $f$ of $H$ assigns a total weight of at least 3 to $a$ and its leaf neighbors. Also, $f$ assigns 
weight at least 3 to $c$ and its leaf neighbors. This means that $f$ assigns at most weight 2 to $b$ and $g$. If $f(b)=2$ and $f(g)=0$ or $f(b)=f(g)=1$, then $g$ is not double Roman dominated. If $f(b)=0$ and $f(g)=2$, then either $b$ is over-dominated or $a$ is assigned weight 0 leading to more than weight 3 on $a$ and its leaf neighbors. Thus, $f(b)+f(g) \geq 3$, a contradiction. Hence, $\gamma_{d R}^{p}(H) \geq 9$ and equality follows. 


\section{LITERATURE SURVEY}

In this section, we review some background results to this thesis. These results present the motivation and origin of perfect double Roman domination.

\subsection{Double Roman Domination}

The term domination was first used by Ore in [16]. He observed that for every graph $G$ of order $n, \gamma(G) \leq \alpha(G)$. Since then, much work has been done on variants of domination including Roman domination, Italian domination, 2-domination, total domination and double Roman domination.

Double Roman domination was first introduced in [2]. The relationship between the double Roman domination number of a graph $G$ and its Roman domination number as well as domination number was discussed in detail here. Also, in [2], in a double Roman dominating function of weight $\gamma_{d R}$-function, no vertex needs to be assigned the value 1 . However, this is not the case with perfect double Roman domination as it requires that vertices are not over-dominated. For example, the path

$P_{4}$ has $\gamma_{d R}^{p}\left(P_{n}\right)=5$ and every $\gamma_{d R}^{p}$-function of $P_{4}$ must assign 1 to a leaf. Below are some of the results we intend to use for the purpose of this thesis:

Corollary 3.1. [2] For any nontrivial connected graph $G, \gamma_{R}(G)<\gamma_{d R}(G)<2 \gamma_{R}(G)$.

Proposition 3.2. [1] For the path $P_{n}$ with $n \geq 1$,

$$
\gamma_{d R}\left(P_{n}\right)= \begin{cases}n & \text { if } n \equiv 0 \bmod 3 \\ n+1 & \text { if } n \equiv 1 \text { or } 2 \bmod 3\end{cases}
$$


Proposition 3.3. [1] For the cycle $C_{n}$ with $n \geq 3$,

$$
\gamma_{d R}\left(C_{n}\right)= \begin{cases}n & \text { if } n \equiv 0,2,3,4 \bmod 6 \\ n+1 & \text { if } n \equiv 1 \text { or } 5 \bmod 6\end{cases}
$$

Also, the main result in [2] gives an upper bound for the double Roman domination number of any tree $T$.

Theorem 3.4. [2] If $T$ is a tree of order $n \geq 3$, then $\gamma_{d R}(T) \leq 5 n / 4$.

This result will be useful in proving the upper bound for the perfect double Roman domination number of trees.

\subsection{Perfect Roman Domination}

A perfect Roman dominating function of a graph $G$ is defined in [12] as a function $f: V(G) \rightarrow\{0,1,2\}$ satisfying the condition that every vertex $u$ with $f(u)=0$ is adjacent to exactly one vertex for which $f(v)=2$. The upper bound of the perfect Roman domination number $\gamma_{R}^{p}(T)$ for a tree $T$ of order $n \geq 3$ is $\frac{4}{5} n$ with the family of trees achieving this bound defined below.

Let $\mathcal{F}$ be the family of all trees $T$ whose vertex set can be partitioned into sets, each set inducing a $P_{5}$ on five vertices, such that the subgraph induced by the central vertices of these $P_{5}$ 's is connected. We call the subtree induced by these central vertices the underlying subtree of the resulting tree $T$, and we call each such path $P_{5}$ a base path of the tree $T$. A tree in the family $\mathcal{F}$ with six base paths and whose underlying subtree is a path $P_{6}$ is illustrated below: 


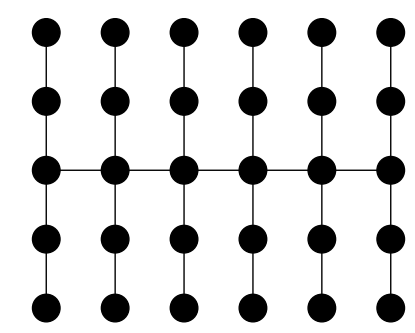

Figure 3: A tree $T \in \mathcal{F}$ 


\section{BASIC RESULTS}

Since every PDRD-function is a double Roman dominating function, we have the following observations.

Observation 4.1. For any graph $G, \gamma_{d R}(G) \leq \gamma_{d R}^{p}(G)$.

Observation 4.2. For any graph $G$ of order $n, \gamma_{d R}^{p}(G) \leq 2 n$ with equality if and only if $G=\bar{K}_{n}$.

Observation 4.3. For any nontrivial graph $G, \gamma_{d R}^{p}(G) \geq 3$.

Proposition 4.4. Let $G$ be a connected nontrivial graph of order $n$. Then $\gamma_{d R}^{p}(G)=3$ if and only if $\Delta(G)=n-1$.

Proof. Suppose $\gamma_{d R}^{p}(G)=3$. Let $f=\left(V_{0}, V_{1}, V_{2}, V_{3}\right)$ be a $\gamma_{d R}^{p}$-function of $G$. Since $f(V)=3$, if $V_{3} \neq \emptyset$, then $\left|V_{3}\right|=1$ and $V_{2}=V_{1}=\emptyset$ with $V_{0}=V-V_{3}$. In this case, let $V_{3}=\{v\}$. Then $v$ is adjacent to all vertices in $V_{0}$ and $\operatorname{deg}(v)=\Delta(G)=n-1$. Hence, we may assume that $V_{3}=\emptyset$, for otherwise the result holds. Then $f(V)=3=2\left|V_{2}\right|$ $+\left|V_{1}\right|$ and this implies that $\left|V_{2}\right| \leq 1$. Thus, $V_{0}=\emptyset$ and $\left|V_{2}\right|=\left|V_{1}\right|=1$ which means $n=2$ and $G \cong P_{2}$ with $\Delta(G)=n-1$. Conversely, suppose $\Delta(G)=n-1$. By Observation 3, we note that $\gamma_{d R}^{p}(G) \geq 3$ for any nontrivial graph $G$. Now, let $v \in V(G)$ such that $\operatorname{deg}(v)=\Delta(G)=n-1$. Then the function $f$ that assigns 3 to $v$ and 0 to other vertices in $G$ is a PDRD-function of $G$ with weight $w(f)=3$. Hence, $\gamma_{d R}^{p}(G) \leq w(f)=3$ and equality follows. 
Corollary 4.5. For complete graphs and stars, $\gamma_{d R}^{p}\left(K_{n}\right)=\gamma_{d R}^{p}\left(K_{1, n-1}\right)=3$.

Proposition 4.6. For the paths $P_{n}$,

$$
\gamma_{d R}^{p}\left(P_{n}\right)= \begin{cases}n & \text { if } n \equiv 0 \bmod 3 \\ n+1 & \text { if } n \equiv 1 \operatorname{or} 2 \bmod 3 .\end{cases}
$$

Proof. Let the vertex set of $P_{n}$ be defined as $V\left(P_{n}\right): u_{1}, u_{2}, \ldots, u_{n}$. We consider three cases based on $n$ :

Case 1: $n \equiv 0 \bmod 3$.

We can partition $P_{n}$ into $P_{3}$ 's. The function $f$ that assigns the weight 3 to the central vertex of each $P_{3}$ and weight 0 to other vertices is a PDRD-function of $P_{n}$ with $w(f)=n$. Thus, $\gamma_{d R}^{p}\left(P_{n}\right) \leq w(f)=n$. By Observation 4.1 and Proposition 3.2, $\gamma_{d R}^{p}\left(P_{n}\right) \geq \gamma_{d R}\left(P_{n}\right)=n$. Hence, $\gamma_{d R}^{p}\left(P_{n}\right) \geq n$ and equality follows.

Case 2: $n \equiv 1 \bmod 3$.

If $n$ is even, say $n=2 k$. Let $f$ be a function that assigns weight 2 to $u_{1}, u_{3}, u_{5}, \ldots, u_{2 k-1}$, the weight 0 to $u_{2}, u_{4}, u_{6}, \ldots, u_{2 k-2}$ and weight 1 to $u_{2 k}$. Then, $f$ is a PDRD-function of $P_{n}$ with $w(f)=\frac{2 n}{2}+1=n+1$. Hence, $\gamma_{d R}^{p}\left(P_{n}\right) \leq w(f)=n+1$. If $n$ is odd, say $n=2 k+1$, then the function $f$ that assigns the weight 2 to $u_{1}, u_{3}, u_{5}, \ldots, u_{2 k+1}$ and weight 0 to all other vertices is a PDRD-function of the $P_{n}$ with $w(f)=\frac{2(n+1)}{2}=$ $n+1$. Thus, $\gamma_{d R}^{p}\left(P_{n}\right) \leq w(f)=n+1$. By Observation 4.1 and Proposition 3.2, $\gamma_{d R}^{p}\left(P_{n}\right) \geq \gamma_{d R}\left(P_{n}\right)=n+1$. Hence, $\gamma_{d R}^{p}\left(P_{n}\right) \geq n+1$ and equality follows.

Case 3: $n \equiv 2 \bmod 3$.

Suppose $n=3 k+2$. Let $f$ be a function that assigns weight 3 to $u_{2}, u_{5}, u_{8}, \ldots, u_{3 k-1}, u_{3 k+2}$, the weight 0 to all other vertices. Then, $f$ is a PDRD-function of $P_{n}$ with $w(f)=$ 
$\frac{3(n-2)}{3}+3=n+1$. Hence, $\gamma_{d R}^{p}\left(P_{n}\right) \leq w(f)=n+1$. By Observation 4.1 and Proposition 3.2, $\gamma_{d R}^{p}\left(P_{n}\right) \geq \gamma_{d R}\left(P_{n}\right)=n+1$. Hence, $\gamma_{d R}^{p}\left(P_{n}\right) \geq n+1$ and equality follows.

Proposition 4.7. For the cycles $C_{n}$,

$$
\gamma_{d R}^{p}\left(C_{n}\right)= \begin{cases}n & \text { if } n \equiv 0,2,3,4 \bmod 6 \\ n+1 & \text { if } n \equiv 1 \text { or } 5 \bmod 6\end{cases}
$$

Proof. Let the vertex set of the $C_{n}$ be defined as $V\left(C_{n}\right): u_{1}, u_{2}, u_{3}, \ldots, u_{n-1}, u_{n}$. We consider these cases based on $n$.

Case 1: $n \equiv 0,2,4 \bmod 6$.

The function $f$ that assigns the weight 2 to $u_{1}, u_{3}, u_{5}, \ldots, u_{n-1}$ and weight 0 to other vertices is a PDRD-function of $C_{n}$ with weight $w(f)=2 n / 2=n$. Hence, $\gamma_{d R}^{p}\left(C_{n}\right) \leq$ $w(f)=n$. By Observation 4.1 and Proposition 3.3, $\gamma_{d R}^{p}\left(C_{n}\right) \geq \gamma_{d R}\left(C_{n}\right)=n$. Hence, $\gamma_{d R}^{p}\left(C_{n}\right) \geq n$ and equality follows.

Case 2: $n \equiv 1 \bmod 6$.

Suppose $f$ is a function that assigns weight 2 to $u_{1}, u_{3}, u_{5}, \ldots, u_{n-2}, u_{n}$ and weight 0 to other vertices. Then $f$ is a PDRD function of $C_{n}$ with weight $w(f)=\frac{2(n-1)}{2}+2=$ $n+1$. Hence, $\gamma_{d R}^{p}\left(C_{n}\right) \leq w(f)=n+1$. By Observation 4.1 and Proposition 3.3, $\gamma_{d R}^{p}\left(C_{n}\right) \geq \gamma_{d R}\left(C_{n}\right)=n+1$. Hence, $\gamma_{d R}^{p}\left(C_{n}\right) \geq n+1$ and equality follows.

Case 3: $n \equiv 3 \bmod 6$.

Suppose $f$ is a function that assigns weight 3 to $u_{1}, u_{4}, u_{7}, \ldots, u_{n-2}$ and weight 0 to other vertices. Then $f$ is a PDRD-function of $C_{n}$ with weight $w(f)=3 n / 3=n$. 
Hence, $\gamma_{d R}^{p}\left(C_{n}\right) \leq w(f)=n$. By Observation 4.1 and Proposition 3.3, $\gamma_{d R}^{p}\left(C_{n}\right) \geq$ $\gamma_{d R}\left(C_{n}\right)=n$. Hence, $\gamma_{d R}^{p}\left(C_{n}\right) \geq n$ and equality follows.

Case $4: n \equiv 5 \bmod 6$.

The function $f$ that assigns the weight 2 to $u_{1}, u_{3}, u_{5}, \ldots, u_{n-2}, u_{n}$ and weight 0 to other vertices is a PDRD function of $C_{n}$ with weight $w(f)=\frac{2(n-1)}{2}+2=n+1$. Hence, $\gamma_{d R}^{p}\left(C_{n}\right) \leq w(f)=n+1$. By Observation 4.1 and Proposition 3.3, $\gamma_{d R}^{p}\left(C_{n}\right) \geq$ $\gamma_{d R}\left(C_{n}\right)=n+1$. Hence, $\gamma_{d R}^{p}\left(C_{n}\right) \geq n+1$ and equality follows.

Proposition 4.8. For the double star $S_{r, s}$,

$$
\gamma_{d R}^{p}\left(S_{r, s}\right)= \begin{cases}5 & \text { if } 1=r=s \\ 6 & \text { otherwise }\end{cases}
$$

Proof. Let $u$ and $v$ be non-leaves in $S_{r, s}$ and suppose $u$ is adjacent to $r$ leaves and $v$ is adjacent to $s$ leaves. We consider three cases based on $r$ and $s$ :

Case 1: $1=r=s$

In this case, $S_{r, s} \cong P_{4}$ and $\gamma_{d R}^{p}\left(P_{4}\right)=5$.

Case 2: $S_{r, s} ¥ P_{4}$

First, we consider the case where $1=r<s$. In this case, the function $f$ that assigns weight 3 to each of $u$ and $v$ and weight 0 to other vertices is a PDRD-function of $S_{r, s}$ with weight $w(f)=6$. Hence, $\gamma_{d R}^{p}\left(S_{r, s}\right) \leq w(f)=6$. Now, we claim that $\gamma_{d R}^{p}\left(S_{r, s}\right) \geq 6$. Suppose to the contrary that $\gamma_{d R}^{p}\left(S_{r, s}\right) \leq 5$. To perfectly double Roman dominate $v$ and its leaf neighbors, we need at least weight 3 . This means that we have to use weight of at most 2 on $u$ and its leaf neighbor, say $r$. If $f(u)=2$ and 
$f(r)=0$ or if $f(u)=f(r)=1$, then $r$ is not perfectly double Roman dominated. Also, if $f(u)=0$ and $f(r)=2$, then either $u$ is over-dominated or $v$ is assigned 2, implying that $v$ and its leaf neighbors have total weight at least 4 . Hence, $f(u)+f(r) \geq 3$, a contradiction. Thus, $\gamma_{d R}^{p}\left(S_{r, s}\right) \geq 6$ and equality follows. Next, we consider the case where $2 \leq r \leq s$. In this case, the function $f$ that assigns weight 3 to each of $u$ and $v$ and weight 0 to other vertices is a PDRD-function with weight $w(f)=6$. Hence, $\gamma_{d R}^{p}\left(S_{r, s}\right) \leq w(f)=6$. Now, we claim that $\gamma_{d R}^{p}\left(S_{r, s}\right) \geq 6$. Suppose to the contrary that $\gamma_{d R}^{p}\left(S_{r, s}\right) \leq 5$. To perfectly double Roman dominate $v$ and its leaf neighbors, we need at least weight 3 . This means that we have to use at most weight 2 on $u$ and its leaf neighbors. If $f(u)=2$, then its leaf neighbors are not perfectly double Roman dominated. Also, if $f(u)=0$, and we assign 2 to one of its leaf neighbors, then the other leaf neighbors of $u$ are not dominated. Hence, we need at least 3 to perfectly double Roman dominate $u$ and its leaf neighbors, which is a contradiction. Thus, $\gamma_{d R}^{p}\left(S_{r, s}\right) \geq 6$ and equality follows. 


\section{MAIN RESULTS}

In this section, we present a family of trees where $\gamma_{d R}^{p}(T)>\gamma_{d R}(T)$. Further, we prove that for any tree $T$ of order $n, \gamma_{d R}^{p}(T) \leq 3 n / 2$.

Proposition 5.1. Let $T$ be a tree of order $n$. If there exists three vertices $u, v$ and $w$ such that $G[\{u, v, w\}]=P_{3}$ where $w$ is adjacent to exactly one leaf or $\operatorname{deg}(w)=2$ and $f(u)=f(v)=3$ for every $\gamma_{d R}$-function and $\gamma_{d R}^{p}$-function of $T$, then $\gamma_{d R}^{p}(T)>\gamma_{d R}(T)$.

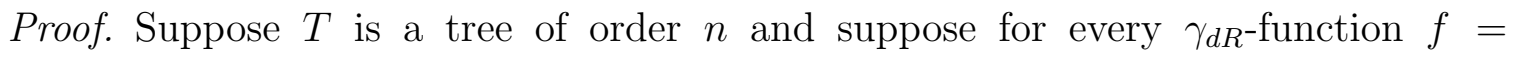
$\left(V_{0}, V_{1}, V_{2}, V_{3}\right)$ of $T$, there exists $u, v \in V_{3}$ such that $d(u, v)=2$ and the $u v$ path in $T$ contains vertex $w$ where $w$ is adjacent to exactly one leaf. In this case, since $w$ is adjacent to only one leaf, say $x$, then $f$ assigns weight 2 to $x$ to double Roman dominate $x$. However, this is not the case for perfect double Roman domination as it leads to over-domination of $w$. Hence, to perfectly double Roman dominate $w$ and $x$, we assign total weight 3 to $w$ and $x$. This is attained either by assigning $w$ weight 3 and $x$ weight 0 or assigning $w$ weight 2 and $x$ weight 1 . Hence, $\gamma_{d R}^{p}(T)>\gamma_{d R}(T)$ in this case. Now, we may assume $\operatorname{deg}(w)=2$. In this case, $f$ assigns weight 0 to $w$. Again, this is not the case for perfect double Roman domination and any $\gamma_{d R}^{p}$-function assigns weight 2 to $w$. Thus, $\gamma_{d R}^{p}(T)>\gamma_{d R}(T)$.

Now we show that the family of trees of order $n$ attaining the upper bound of $5 n / 4$ on $\gamma_{d R}(T)$ in [2] also has $\gamma_{d R}^{p}(T)=5 n / 4$. We define the family $\mathcal{T}$ of trees as follows:

Let $T^{\prime}$ be an arbitrary tree of order $n^{\prime} \geq 1$, and let $T$ be the tree of order $n=4 n^{\prime}$ obtained from $T^{\prime}$ by adding to each vertex of $T^{\prime}$ three new vertices $v_{1}, v_{2}, v_{3}$ and edges 


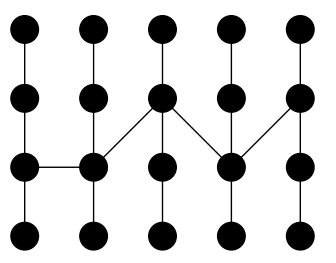

Figure 4: A tree $T \in \mathcal{T}$

$v v_{1}, v v_{2}$ and $v_{2} v_{3}$. Then $v_{1}$ is a leaf neighbor of $v$, while $v_{2}$ is a neighbor of $v$ with degree 2 and $v_{3}$ is a leaf neighbor of $v_{2}$. Note that $\mathcal{T}$ contains $P_{4}$. We call $T^{\prime}$ the underlying subtree of $T$. For each $v \in V\left(T^{\prime}\right)$, we call the $P_{4}=\left(v_{1}, v, v_{2}, v_{3}\right)$ a base path. An example of a tree $T$ in the family $\mathcal{T}$ whose underlying subtree is a path $P_{5}$ is illustrated in Figure 4.

Lemma 5.2. If $T$ is a tree of order $n$ that belongs to $\mathcal{T}$ with underlying tree $T^{\prime}$, then the following hold:

(a) $\gamma_{d R}^{p}(T)=5 n / 4$

(b) There exists a $\gamma_{d R}^{p}$-function that assigns the weight 0 to every vertex in the underlying tree $T^{\prime}$. Further, there exists a $\gamma_{d R}^{p}$-function of $T$ that assigns weight of 2 to every vertex in the underlying tree $T^{\prime}$.

Proof. Let $T \in \mathcal{T}$ have order $n$, and let the underlying subtree $T^{\prime}$ of $T$ have order $k$ and so $n=4 k$ (with $k \geq 1$ ). We claim that the sum of the weights on any base path is at least 5. To see this, let $f$ be an arbitrary $\gamma_{d R^{p}}^{p}$ function of $T$ and suppose $P: v_{1} v v_{2} v_{3}$ is an arbitrary base path of $T$. Then $v_{1}$ and $v_{3}$ are leaves in $T$. Suppose to the contrary, that the weight of $f$ on $P$ is at most 4 .

If $f\left(v_{1}\right)=0$, then $f(v)=3$ leaving exactly one of $v_{2}$ and $v_{3}$ to be assigned 1 and the 
other assigned weight 0 . If $f\left(v_{2}\right)=0$ and $f\left(v_{3}\right)=1$, then $v_{3}$ is not double Roman dominated. Similarly, if $f\left(v_{2}\right)=1$ and $f\left(v_{3}\right)=0$, then neither $v_{2}$ nor $v_{3}$ is perfectly double Roman dominated. Hence, $f\left(v_{1}\right) \geq 1$. If $f\left(v_{1}\right)=1$, then $f(v)=2$ to perfectly double Roman dominate $v_{1}$. Then, as before, either $v_{3}$ or both $v_{2}$ and $v_{3}$ are not perfectly double Roman dominated in this case. Hence, $f\left(v_{1}\right) \geq 2$. But there is no way to perfectly double roman dominate both $v_{2}$ and $v_{3}$ using total weight of at most 2 on $v, v_{2}$ and $v_{3}$. Thus, the sum of the weight on any arbitrary base path is at least 5 . Since there are $k$ such (vertex-disjoint) base paths in $T$, each of which receives a total weight of at least 5 under $f$, the weight of $f$ is $w(f) \geq 5 k$. Since $f$ is an arbitrary $\gamma_{d R}^{p}$-function of $T$, this implies that $\gamma_{d R}^{P}(T) \geq 5 k=5 n / 4$. The function $g$ that assigns the weight 2 to vertex $v$ in the base path $P$ defined above, the weight 1 to its leaf neighbor $v_{1}$, weight 2 to $v_{3}$, and weight 0 to $v_{2}$ is a PDRD-function of weight 5 on $P$. Since there are $k$ such (vertex-disjoint) base paths in $T$, each of which receives a total weight of 5 under $g$, then $w(g)=5 k$. Thus, $\gamma_{d R}^{P}(T) \leq w(g)=5 k=5 n / 4$. This proves (a).

Clearly, the function $g$ defined above applied to each base path is a $\gamma_{d R}^{P}(T)$-function of $T$ that assigns 2 to every vertex of the underlying tree. Further, we note that the function $h$ that assigns the weight 0 to $v$, weight 2 to $v_{1}$ and $v_{2}$, and weight 1 to $v_{3}$ to each base path in $T$ is a $\gamma_{d R}^{p}$-function of $T$ that assigns weight 0 to every vertex of the underlying tree. This proves (b).

However, there are trees having $\gamma_{d R}^{p}(T)>5 n / 4$. For example, the tree $T$ in Figure 6 has $\gamma_{d R}^{p}(T) \approx 9 n / 7$. We next show that $3 n / 2$ is an upper bound for $\gamma_{d R}^{p}(T)$ for trees 
of order $n$. Note that a rooted tree $T$ is a tree with distinguished vertex $r$ called the root. In a rooted tree, the parent of a vertex is the vertex connected to it on the path to the root; every vertex except the root has a unique parent. A child of a vertex $v$ is a vertex of which $v$ is the parent. In a rooted tree, all vertices have at most one parent. Also, for a tree $T$ rooted at a vertex $r$ and a vertex $v \neq r$ of $T$, let $T_{v}$ denote the subtree rooted at $v$, consisting of $v$ and its descendants in $T$. Further, let $T-T_{v}$ denote the tree rooted at $r$ formed by removing the subtree $T_{v}$ from $T$. Note that the vertices and edges of $T_{v}$ and the edge from $v$ to its parent in $T$ are removed from $T$ to form $T-T_{v}$ and that $T-T_{v}$ is a tree.

Theorem 5.3. For any tree $T$ of order $n \geq 3, \gamma_{d R}^{p}(T) \leq 3 n / 2$.

Proof. We proceed by induction on the order $n \geq 3$ of a tree $T$. If $n=3$, then $T \cong P_{3}$ and $\gamma_{d R}^{p}(T)=3<3 n / 2$. This establishes the base case. Let $n \geq 4$ and assume that if $T^{\prime}$ is a tree of order $n^{\prime}$, where $n^{\prime}<n, \gamma_{d R}^{p}\left(T^{\prime}\right) \leq 3 n^{\prime} / 2$. Let $T$ be a tree of order $n$. If $\operatorname{diam}(T)=2$, then $T$ is a star. Hence, the function $f$ that assigns weight 3 to the central vertex and weight 0 to every leaf of the star is a PDRD-function of $T$ with weight $w(f)=3$. So, $\gamma_{d R}^{p}(T) \leq 3<3 n / 2$. Hence, we may assume $\operatorname{diam}(T) \geq 3$. Suppose $\operatorname{diam}(T)=3$, then $T$ is a double star $S_{r, s}$ where $r \geq s \geq 1$. Suppose $u$ and $v$ are vertices of $T$ that are not leaves, where $u$ has $r$ leaf neighbors and $v$ has $s$ leaf neighbors. If $r=s=1$, then $T \cong P_{4}$ and $\gamma_{d R}^{p}(T)=5<3 n / 2$. Also, if $r>s=1$, then by Proposition 4.8, $\gamma_{d R}^{p}(T)=6<3 n / 2$. Further, if $r \geq s>1$, then by Proposition $4.8, \gamma_{d R}^{p}(T)=6<3 n / 2$. Hence, we may assume that $\operatorname{diam}(T) \geq 4$.

Let $u$ and $r$ be two vertices at maximum distance apart in $T$. Necessarily, $u$ and $r$ are leaves in $T$ and $d(u, r)=\operatorname{diam}(T)$. We now root the tree $T$ at the vertex $r$. Let 
$v$ be the parent of $u, w$ the parent of $v, x$ the parent of $w$ and let $y$ be the parent of $x$. We note that if $\operatorname{diam}(T)=4$, then $y=r$, otherwise $y \neq r$.

Claim 1. If $\operatorname{deg}(v) \geq 5$, then $\gamma_{d R}^{p}(T)<3 n / 2$.

Proof. Suppose that $\operatorname{deg}(v) \geq 5$. Let $T^{\prime}$ be the tree obtained from $T$ by deleting $u$. Therefore, $v$ is a strong support vertex in $T^{\prime}$ and $n^{\prime} \geq 3$. Applying the inductive hypothesis to $T^{\prime}$, we have $\gamma_{d R}^{p}\left(T^{\prime}\right) \leq 3 n^{\prime} / 2$. Let $f^{\prime}$ be a $\gamma_{d R^{p}}^{p}$-function of $T^{\prime}$. If $f^{\prime}(v)=3$, we can form a PDRD-function $f$ of $T$ by letting $f(z)=f^{\prime}(z)$ for all $z \in T^{\prime}$ and assign weight 0 to $u$. Thus, $\gamma_{d R}^{p}(T) \leq w(f)=w\left(f^{\prime}\right) \leq 3 n^{\prime} / 2=3(n-1) / 2<3 n / 2$. Hence, we may assume that $f^{\prime}(v) \leq 2$ for otherwise, the desired result holds. If $f^{\prime}(v)=2$, then we can form a PDRD-function $f$ of $T$ by letting $f(z)=f^{\prime}(z)$ for all $z \in T^{\prime}$ and assign weight 1 to $u$. Thus, $\gamma_{d R}^{p}(T) \leq w(f)=w\left(f^{\prime}\right)+1 \leq 3 n^{\prime} / 2+1=3(n-1) / 2+1<3 n / 2$. Now, we may assume that $f^{\prime}(v) \leq 1$. In this case, the leaf neighbors of $v$ in $T^{\prime}$ would each have to be assigned weight 2 or 3 which leads to over-domination of $v$. This implies that $f^{\prime}$ is not a PDRD-function of $T^{\prime}$ and we are finished.

Claim 2. If $\operatorname{deg}(v)=4$, then $\gamma_{d R}^{p}(T)<3 n / 2$.

Proof. Let $T^{\prime}$ be the tree obtained from $T$ as follows: delete $v$ and the three leaf children of $v$ and add three new vertices, $w_{1}, w_{2}$ and $w_{3}$ and new edges $w w_{1}, w w_{2}$, and $w_{2} w_{3}$. Then $n^{\prime}=n-4+3=n-1 \geq 6$. Applying the inductive hypothesis to $T^{\prime}$, we have that $\gamma_{d R}^{p}\left(T^{\prime}\right) \leq 3 n^{\prime} / 2=3(n-1) / 2$. Let $f^{\prime}$ be a $\gamma_{d R^{-}}^{p}$ function of $T^{\prime}$. Notice that if $f^{\prime}(w) \geq 2$, then a total weight of 3 is assigned to the vertices $w_{1}, w_{2}$, and $w_{3}$. On the other hand, if $f^{\prime}(w) \leq 1$, then a total weight of 5 is assigned to the vertices $w_{1}, w_{2}$, and $w_{3}$. If $f^{\prime}(w) \geq 2$, then the function $f$ of $T$ that assigns 3 
to $v, 0$ to each leaf child of $v$, and $f(z)=f^{\prime}(z)$ for all $z \in V\left(T^{\prime}\right) \backslash\left\{w_{1}, w_{2}, w_{3}\right\}$ is a PDRD-function of $T$. Thus, $\gamma_{d R}^{p}(T) \leq 3(n-1) / 2+3-3<3 n / 2$. If $f^{\prime}(w)=1$, then it follows that $f^{\prime}\left(w_{1}\right)=2$ and all other neighbors of $w$ in $T^{\prime}$ are assigned 0 or 1 under $f^{\prime}$. Hence, the function $f$ of $T$ that assigns 2 to $v, 1$ to each leaf child of $v$, and $f(z)=f^{\prime}(z)$ for all $z \in V\left(T^{\prime}\right) \backslash\left\{w_{1}, w_{2}, w_{3}\right\}$ is a PDRD-function of $T$. Thus, $\gamma_{d R}^{p}(T) \leq 3(n-1) / 2+5-5<3 n / 2$. If $f^{\prime}(w)=0$, then one of the following holds: $f^{\prime}\left(w_{1}\right)=3$ and all other neighbors of $w$ in $T^{\prime}$ are assigned 0 or 1 under $f^{\prime}$, or $f^{\prime}\left(w_{1}\right)=2, f^{\prime}\left(w_{2}\right)=2$, and all other neighbors of $w$ in $T^{\prime}$ are assigned 0 or 1 under $f^{\prime}$, or $f^{\prime}\left(w_{1}\right)=2, f^{\prime}(x)=2$, and all other neighbors of $w$ in $T^{\prime}$ are assigned 0 or 1 under $f^{\prime}$. If $f^{\prime}\left(w_{1}\right)=3$, then function $f$ of $T$ that assigns 3 to $v, 0$ to each leaf child of $v$, and $f(z)=f^{\prime}(z)$ for all $z \in V\left(T^{\prime}\right) \backslash\left\{w_{1}, w_{2}, w_{3}\right\}$ is a PDRD-function of $T$. Thus, $\gamma_{d R}^{p}(T) \leq 3(n-1) / 2+3-5<3 n / 2$. If $f^{\prime}\left(w_{1}\right)=2$ and $f^{\prime}\left(w_{2}\right)=2$, then again the function $f$ of $T$ that assigns 3 to $v, 0$ to each leaf child of $v$, and $f(z)=f^{\prime}(z)$ for all $z \in V\left(T^{\prime}\right) \backslash\left\{w_{1}, w_{2}, w_{3}\right\}$ is a PDRD-function of $T$. Thus, $\gamma_{d R}^{p}(T) \leq 3(n-1) / 2+3-5<$ $3 n / 2$. If $f^{\prime}\left(w_{1}\right)=2$ and $f^{\prime}(x)=2$, then the function $f$ of $T$ that assigns 2 to $v, 1$ to each leaf child of $v$, and $f(z)=f^{\prime}(z)$ for all $z \in V\left(T^{\prime}\right) \backslash\left\{w_{1}, w_{2}, w_{3}\right\}$ is a PDRDfunction of $T$. Thus, $\gamma_{d R}^{p}(T) \leq 3(n-1) / 2+5-5<3 n / 2$.

Claim 3. If $\operatorname{deg}(v)=3$, then $\gamma_{d R}^{P}(T)<3 n / 2$.

Proof. If $\operatorname{deg}(v)=3$, then $v$ has exactly two leaf children in $T$. Let $T^{\prime}=T-u$, then $n^{\prime} \geq 3$. Applying the inductive hypothesis to $T^{\prime}$, we have $\gamma_{d R}^{p}\left(T^{\prime}\right) \leq 3 n^{\prime} / 2$. Suppose $f^{\prime}$ is a $\gamma_{d R^{-}}^{p}$ function of $T^{\prime}$. If $f^{\prime}(v)=3$, we can form a PDRD-function $f$ of $T$ by letting $f(z)=f^{\prime}(z)$ for all $z \in T^{\prime}$ and assign weight 0 to $u$. Thus, $\gamma_{d R}^{p}(T) \leq w(f)=w\left(f^{\prime}\right) \leq 3 n^{\prime} / 2=3(n-1) / 2<3 n / 2$. If $f^{\prime}(v)=2$, then we can 
form a PDRD-function $f$ of $T$ by letting $f(z)=f^{\prime}(z)$ for all $z \in T^{\prime}$ and assign weight 1 to $u$. Thus, $\gamma_{d R}^{p}(T) \leq w(f)=w\left(f^{\prime}\right)+1 \leq 3 n^{\prime} / 2+1=3(n-1) / 2+1<3 n / 2$. Now, we may assume that $f^{\prime}(v) \leq 1$ for otherwise, the result holds. If $f^{\prime}(v)=1$, then the leaf neighbor of $v$ in $T^{\prime}$ is assigned weight 2 and $f^{\prime}(w) \leq 1$, for otherwise $v$ will be over-dominated. Therefore, we can form a PDRD-function $f$ on $T$ by letting $f(z)=f^{\prime}(z)$ for all $z \in T^{\prime}-v$, reassign weight 0 to $v$ and assign weight 2 to $u$. Thus, $\gamma_{d R}^{p}(T) \leq w(f)=w\left(f^{\prime}\right)+1 \leq 3 n^{\prime} / 2+1=3(n-1) / 2+1<3 n / 2$. If $f^{\prime}(v)=0$, then we consider two cases based on the weight assigned to the leaf child of $v$ in $T^{\prime}$, say $t$. Note that if $f^{\prime}(v)=0$, then $f^{\prime}(t) \in\{2,3\}$. If $f^{\prime}(t)=3$, then $f^{\prime}(w) \leq 1$ and we can form a PDRD-function $f$ of $T$ by letting $f(z)=f^{\prime}(z)$ for all $z \in T^{\prime}-t$ and assign weight 2 to each of $t$ and $u$. Hence, $\gamma_{d R}^{p}(T) \leq w(f)=w\left(f^{\prime}\right)+1 \leq 3 n^{\prime} / 2+1=3(n-1) / 2+1<$ $3 n / 2$. Now, if $f^{\prime}(t)=2$, then $f^{\prime}(w)=2$ to ensure that $v$ is perfectly double Roman dominated. We can form a PDRD-function $f$ on $T$ by letting $f(z)=f^{\prime}(z)$ for all $z \in T^{\prime}-\{v, t\}$, reassign weight 3 to $v$ and assign weight 0 to both $t$ and $u$. Thus, $\gamma_{d R}^{p}(T) \leq w(f)=w\left(f^{\prime}\right)+1 \leq 3 n^{\prime} / 2+1=3(n-1) / 2+1<3 n / 2$.

Henceforth, we may assume that $\operatorname{deg}(v)=2$.

Claim 4. If $\operatorname{deg}(v)=2$ and $\operatorname{deg}(w)=2$, then $\gamma_{d R}^{P}(T)<3 n / 2$.

Proof. Let $T^{\prime}=T-T_{w}$. Since $\operatorname{diam}(T) \geq 4, T^{\prime}$ has order $n^{\prime} \geq 2$. If $n^{\prime}=2$, then $T \cong P_{5}$ and $\gamma_{d R}^{p}(T)=6<15 / 2=3 n / 2$. Hence, we may assume that $n^{\prime} \geq 3$ and we can apply the inductive hypothesis to $T^{\prime}$. Thus, $\gamma_{d R}^{p}\left(T^{\prime}\right) \leq 3 n^{\prime} / 2$. Recall that $x$ is the parent of $w$. Suppose $f^{\prime}$ is a $\gamma_{d R^{-}}^{p}$-function of $T^{\prime}$. If $f^{\prime}(x)=3$, then we can form a PDRD-function $f$ of $T$ by letting $f(z)=f^{\prime}(z)$ for all $z \in T^{\prime}$, assign weight 0 to 


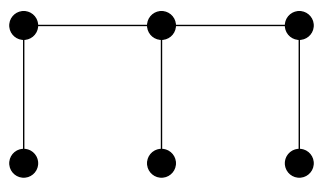

Figure 5: Tree $H_{3}$

both $w$ and $v$, and weight 3 to $u$. Thus, $\gamma_{d R}^{p}(T) \leq w(f)=w\left(f^{\prime}\right)+3 \leq 3 n^{\prime} / 2+3=$ $3(n-3) / 2+3<3 n / 2$. Now, we may assume that $f^{\prime}(x) \leq 2$. If $f^{\prime}(x)=2$, then we can form a PDRD-function $f$ of $T$ by letting $f(z)=f^{\prime}(z)$ for all $z \in T^{\prime}$, assign weight 0 to $w$, weight 2 to $v$ and weight 1 to $u$. Thus, $\gamma_{d R}^{p}(T) \leq w(f)=w\left(f^{\prime}\right)+3 \leq 3 n^{\prime} / 2+3=$ $3(n-3) / 2+3<3 n / 2$. If $f^{\prime}(x) \leq 1$, then we can form a PDRD-function $f$ of $T$ by letting $f(z)=f^{\prime}(z)$ for all $z \in T^{\prime}$, assign weight 0 to both $w$ and $u$, and weight 3 to $v$. Thus, $\gamma_{d R}^{p}(T) \leq w(f)=w\left(f^{\prime}\right)+3 \leq 3 n^{\prime} / 2+3=3(n-3) / 2+3<3 n / 2$.

We now complete the proof of the theorem. Henceforth, we may assume that $\operatorname{deg}(v)=2$ and $\operatorname{deg}(w) \geq 3$, for otherwise the result holds.

Claim 5. If $\operatorname{deg}(v)=2$ and $\operatorname{deg}(w) \geq 3$, then $\gamma_{d R}^{P}(T) \leq 3 n / 2$.

Proof. By our choice of $u$, every child of $w$ is either a leaf or a support vertex of degree 2 ( $\operatorname{similar}$ to $v$ ). Let $s$ be the number of leaf children of $w$ and $t$ be the number of degree 2 children of $w$. Since $\operatorname{deg}(v)=2$ and $\operatorname{deg}(w) \geq 3$, it follows that $t \geq 1$ and $s+t \geq 2$. Let $T^{\prime}$ be the tree obtained from $T$ by removing all the descendants of $w$. Note that $w$ is a leaf in $T^{\prime}$ and $n^{\prime}=n-s-2 t \geq 3$. Applying the inductive hypothesis to $T^{\prime}$, we have that $\gamma_{d R}^{p}\left(T^{\prime}\right) \leq 3 n^{\prime} / 2=3(n-s-2 t) / 2=(3 n-3 s-6 t) / 2$. Let $f^{\prime}$ be a $\gamma_{d R^{p}}^{p}$ function of $T^{\prime}$. If $f^{\prime}(w)=2$, then the function $f$ of $T$ that assigns 1 
to each leaf child of $w, 0$ to each degree 2 child of $w, 2$ to each leaf vertex distance 2 from $w$, and $f(z)=f^{\prime}(z)$ for all $z \in V\left(T^{\prime}\right)$ is a PDRD-function of $T$. Thus, $\gamma_{d R}^{p}(T) \leq$ $(3 n-3 s-6 t) / 2+s+2 t=(3 n-s-2 t) / 2<3 n / 2$. Assume that $f^{\prime}(w)=3$. It follows that $f^{\prime}(x) \neq 1$. Furthermore, $f^{\prime}(x)=0$, otherwise the weight on $w$ can be reduced. Thus, the function $f$ of $T$ that reassigns 1 to $x$ and 2 to $w$, and assigns 1 to each leaf child of $w, 0$ to each degree 2 child of $w, 2$ to each leaf vertex distance 2 from $w$, and $f(z)=f^{\prime}(z)$ for all $z \in V\left(T^{\prime}\right) \backslash\{x, w\}$ is a PDRD-function of $T$. Hence, again, $\gamma_{d R}^{p}(T) \leq(3 n-3 s-6 t) / 2+s+2 t=(3 n-s-2 t) / 2<3 n / 2$. If $f^{\prime}(w)=1$, then $f^{\prime}(x)=2$ to double Roman dominate $w$. The function $f$ of $T$ that reassigns 2 to $w$ and and assigns 1 to each leaf child of $w, 0$ to each degree 2 child of $w, 2$ to each leaf vertex distance 2 from $w$, and $f(z)=f^{\prime}(z)$ for all $z \in V\left(T^{\prime}\right) \backslash\{w\}$ is a PDRD-function of $T$. Hence, again, $\gamma_{d R}^{p}(T) \leq(3 n-3 s-6 t) / 2+1+s+2 t=(3 n-s-2 t+2) / 2$. Thus, $\gamma_{d R}^{p}(T) \leq 3 n / 2$ if $s+2 t \geq 2$. If $f^{\prime}(w)=0$, then $f^{\prime}(x)=3$ to double Roman dominate $w$. The function $f$ of $T$ that reassigns 2 to $w$ and and assigns 1 to each leaf child of $w, 0$ to each degree 2 child of $w, 2$ to each leaf vertex distance 2 from $w$, and $f(z)=f^{\prime}(z)$ for all $z \in V\left(T^{\prime}\right) \backslash\{w\}$ is a PDRD-function of $T$. Hence, again, $\gamma_{d R}^{p}(T) \leq(3 n-3 s-6 t) / 2+2+s+2 t=(3 n-s-2 t+4) / 2$. Thus, $\gamma_{d R}^{p}(T) \leq 3 n / 2$ if $s+2 t \geq 4$. In all cases, the result holds if $s+2 t \geq 4$. Hence, we may assume that $s+2 t \leq 3$. Since $s+t \geq 2, t \geq 1$, and $s+2 t \leq 3$, the only possibility for $s$ and $t$ is if $s=t=1$.

In this case, $T_{w}$ is a path $P_{4}$. Let $T^{\prime}=T-T_{w}$. Then $n^{\prime}=n-4$. If $n^{\prime}=2$, then $T$ is the tree $H_{3}$ (the corona of a $P_{3}$ ). But then $n=6$ and $\gamma_{d R}^{p}(T)=7$, so $\gamma_{d R}^{p}(T)<3 n / 2$. Therefore, we may assume that $n^{\prime} \geq 3$ and applying our inductive 
hypothesis to $T^{\prime}$, we have $\gamma_{d R}^{p}\left(T^{\prime}\right) \leq 3 n^{\prime} / 2=3(n-4) / 2=(3 n-12) / 2$. Let $f^{\prime}$ be a $\gamma_{d R}^{p}$-function of $T^{\prime}$. If $f^{\prime}(x) \geq 2$, then the function $f$ of $T$ that assigns 2 to $w, 1$ to the leaf child of $w, 0$ to $v, 2$ to $u$, and $f(z)=f^{\prime}(z)$ for all $z \in V\left(T^{\prime}\right)$ is a PDRD-function of $T$. Thus, $\gamma_{d R}^{p}(T) \leq(3 n-12) / 2+5<3 n / 2$. If $f^{\prime}(x) \leq 1$, then the function $f$ of $T$ that assigns 0 to $w, 2$ to the leaf child of $w, 2$ to $v, 1$ to $u$,and $f(z)=f^{\prime}(z)$ for all $z \in V\left(T^{\prime}\right)$ is a PDRD-function of $T$. Again, $\gamma_{d R}^{p}(T) \leq(3 n-12) / 2+5<3 n / 2$. Hence, the result holds if $s=t=1$.

Note that we have not been able to find extremal trees attaining the bound of Theorem 5.3. In fact, we believe that this bound can be improved to $9 n / 7$. A member of a family of trees achieving this bound is shown in Figure 6 .

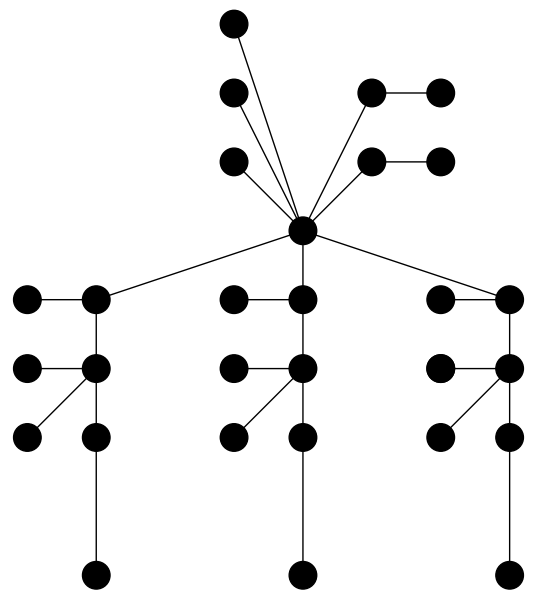

Figure 6: A tree $T$ with $\gamma_{d R}^{p}(T) \approx 9 n / 7$. 


\section{CONCLUDING REMARKS}

We obtained a lower and upper bound of the perfect double Roman domination number for trees. Specifically, we noted that for any nontrivial tree $T$ of order $n$, $3 \leq \gamma_{d R}^{p}(T) \leq 3 n / 2$. We also obtained the exact values of the perfect double Roman domination number for paths, cycles, complete graphs and stars.

\section{Open Problems}

1. Construct a polynomial algorithm to compute the value of $\gamma_{d R}^{p}(T)$ for any tree $T$.

2. Find a family of trees achieving the upper bound. That is, trees with $\gamma_{d R}^{p}(T)=$ $3 n / 2$.

3. Find a relationship between $\gamma_{d R}^{p}(G)$ and $\gamma_{R}^{p}(G)$. 


\section{BIBLIOGRAPHY}

[1] H.A. Ahangar, M. Chellali, S.M. Sheikholeslami, On the double Roman domination in graphs, Discrete Appl. Math. 232 (2017), 1-7.

[2] R.A. Beeler, T.W. Haynes, S.T. Hedetniemi, Double Roman domination, Discrete Appl. Math. 211 (2016), 23-29.

[3] S. Bermudo, H. Fernau, J.M. Sigarreta, The differential and Roman domination of a graph, Appl. Anal. Discrete Math. 8 (2014), 155-171.

[4] E.W. Chambers, B. Kinnersley, N. Prince, D.B. West, Extremal problems for Roman domination, SIAM J. Discrete Math. 23 (2009), 1575-1586.

[5] M. Chellali, T.W. Haynes, S.T. Hedetniemi, A.A. McRae, Roman 2-domination, Discrete Appl. Math. 204 (2016), 22-28.

[6] E.J. Cockayne, P.A. Dreyer Jr., S.M. Hedetniemi, Roman domination in graphs, Discrete Math. 278 (2004), 11-22.

[7] O. Favaron, H. Karami, R. Khoeilar, S.M. Sheikholeslami, On the Roman domination number of a graph, Discrete Math. 309 (2009), 3447-3451.

[8] T.W. Haynes, S.T. Hedetniemi, P.J. Slater Fundamentals of Domination in Graphs, Marcel Dekker, Inc, New York (1998).

[9] T.W. Haynes and M.A. Henning, Perfect Italian Domination, Discrete Math 211 (2018), 4-19. 
[10] M.A. Henning, A characterization of Roman trees, Discuss. Math. Graph Theory 22 (2) (2002), 325-334.

[11] M.A. Henning and S.T. Hedetniemi, Defending the Roman empire - a new strategy, Discrete Math. 266 (2003), 239-251.

[12] M.A. Henning, W.F. Klostermeyer, G.MacGillivray, Perfect Roman domination in trees, Discrete Appl. Math. 236 (2018), 235-245.

[13] W. Klostermeyer, A taxonomy of perfect domination, J. Discrete Math. Sci. Cryptography 18 (2015), 105-116.

[14] C.H. Liu and G.J. Chang, Roman domination on 2-connected graphs, Discrete Math. 26 (2012), 193-205.

[15] C.H. Liu and G.J. Chang, Upper bounds on Roman domination numbers of graphs, Discrete Math. 312 (2012), 1386-1391.

[16] O. Ore, Theory of Graphs, Amer. Math. Soc. Colloq. 38 (1962).

[17] P. Pavlic and J.Zerovnik, Roman domination number of the Cartesian products of paths and cycles, Electron. J. Combin.. 19 (2012) 608-619 .

[18] I. Stewart, Defend the Roman Empire, Sci. Amer. 281 (1999). 


\section{VITA}

\section{AYOTUNDE TOLULOPE EGUNJOBI}

Education:

Professional Experience:
B.S. Mathematics, University of Ilorin Ilorin, Kwara, Nigeria 2013

M.S. Mathematical Sciences, East Tennessee State University Johnson City, Tennessee, USA 2019

Graduate Assistant, FUTMx in Minna Minna, Niger, Nigeria 2013-2014

Research Analyst, Ernst \& Young Lagos, Lagos, Nigeria 2015-2017

Graduate Teaching Assistant, East Tennessee State University Johnson City, Tennessee, USA 2017-2019 